\section{Os Centros de Referência em Saúde do Trabalhador e a notificação de acidentes de trabalho no Brasil}

\author{
Workers' Health Referral Centers and reporting of \\ work-related injuries in Brazil
}

\section{Abstract}

This study examines the contribution of Workers Health Referral Centers (CEREST) to the reporting of severe work-related injuries and those involving exposure to biological materials in the Brazilian National Health Reporting System (SINAN), under the Unified National Health System (SUS). The study used data from the FormSUS and SINAN databases, aggregated for the CEREST coverage areas. Valid data were obtained for 125 CEREST (23 State and 102 regional). A majority of the CEREST were assessed as fully installed. The increase in the reporting of severe work-related accidents was greater when staffing was consistent with the demand, and when teams responded to external demands, including those of the media. For exposures to biological material, CEREST with good physical installations, those that responded to media demands, and those with trained personnel in the sentinel network showed a higher increase in reporting. Infrastructure, staff numbers and training, and responding to external demands are important for increasing notification of work-related accidents and should be prioritized in order to reduce the major underreporting of such accidents.

Occupational Accidents Registry; Occupational Accidents; Occupational Health; Information Systems

\author{
Adriana Galdino 1,2 \\ Vilma Sousa Santana 3 \\ Silvia Ferrite 4
}

\section{Introdução}

Os acidentes de trabalho têm expressiva morbimortalidade, constituindo-se em importante problema de saúde pública. No Brasil, agravos relacionados ao trabalho representam aproximadamente $25 \%$ das lesões por causas externas atendidas em serviços de emergência ${ }^{1}$ e mais de $70 \%$ dos benefícios acidentários da Previdência Social 2 .

Os sistemas de informação em saúde no país são avançados, mas dados sobre acidentes de trabalho continuam a demandar melhores registros, tanto de cobertura, como de qualidade dos dados. Há grande sub-registro do Sistema de Informações de Agravos de Notificação (SINAN) ${ }^{3}$, e os dados mais amplamente utilizados, da Previdência Social, são parciais, restritos a trabalhadores segurados que perfazem apenas um terço da população economicamente ativa ocupada ${ }^{2}$. Dados da Previdência Social são também subregistrados 4,5 .

Com vistas a melhorar a qualidade e ampliar a cobertura do registro, o Sistema Único de Saúde (SUS) incorporou ao SINAN 11 doenças e agravos relacionados ao trabalho, que compõem a lista de prioridades no país 6 . Entre estas, são notificáveis os acidentes considerados graves e os que ocorreram com exposição a material biológico, em sua maioria acidentes pérfurocortantes ${ }^{3}$. Notificações de acidente de trabalho 
grave são indicadas para os casos fatais, os que resultaram em mutilações e aqueles cujas vítimas eram menores de 18 anos. Os acidentes com exposição a material biológico são os que envolvem sangue e outros fluidos orgânicos, ocorrendo comumente com profissionais da saúde. Em 2007 foi iniciada a implantação dessas notificações no SINAN 7 e incluídos conteúdos relativos à saúde do trabalhador nos programas de treinamento respectivos, em especial nos cursos preparatórios das equipes das unidades sentinelas, isto é, serviços de saúde com a responsabilidade de notificar estes agravos específicos. Foram também elaboradas e adotadas normas de procedimentos, organizadas em protocolos e instruções normativas 7 .

A Rede Nacional de Atenção Integral à Saúde do Trabalhador (RENAST) foi instituída pelo SUS em 20026 . Conta com uma rede de informações e práticas de saúde organizada para realizar ações assistenciais, de vigilância e de promoção da saúde; compreende Centros de Referência em Saúde do Trabalhador (CEREST), estaduais, regionais e municipais, as unidades sentinelas, núcleos de saúde do trabalhador e demais serviços do SUS voltados para esse campo de atuação em saúde. Os CEREST, no âmbito de um determinado território, são polos irradiadores do conhecimento sobre a relação processo de trabalho/processo saúde-doença, com responsabilidade de oferecer suporte técnico e científico às demais unidades do SUS, em especial às da atenção básica. Esses Centros detêm a responsabilidade de notificar, investigar e dar respostas sensíveis aos agravos relacionados ao trabalho, visando ao controle e melhoria das condições deste 6 .

De acordo com dados da Coordenação Geral de Saúde do Trabalhador (CGSAT), do Ministério da Saúde, em 2008, mais da metade das Unidades Federadas (UF) notificavam acidentes de trabalho graves e aqueles com exposição a material biológico, apesar de haver problemas com a qualidade dos registros, sendo comuns dados faltantes ${ }^{3}$. Entre 2007 e 2008, verificou-se aumento expressivo da notificação de ambos os agravos na maioria dos estados e regiões ${ }^{3}$. Em 2007, foram notificados 18.477 casos de acidentes de trabalho graves; em 2008, 29.629, o que corresponde a um aumento de $60,4 \%$ no Brasil. Notificações para acidentes de trabalho com exposição a material biológico foram 13.749, em 2007, e 20.418, em 2008, crescimento de 48,5\% 3. Embora comparações sejam limitadas pela não uniformidade de definições operacionais, registros do Instituto Nacional do Seguro Social (INSS), restritos a trabalhadores segurados, representaram, em 2007, 493.349 casos de acidentes de trabalho que cursaram com incapacidade para o trabalho por mais de 15 dias ${ }^{2}$, demonstrando a extensa subnotificação do SINAN.

Não foram encontrados estudos sobre causas do sub-registro ou subnotificação de acidentes de trabalho conduzidos no Brasil. Entretanto, sabendo-se que há crescimento da notificação de acidentes de trabalho graves ou acidentes de trabalho com exposição a material biológico, o conhecimento dos fatores impulsionadores da notificação pode contribuir para o desenvolvimento de ações mais apropriadas e direcionadas para os pontos frágeis da RENAST e CEREST, visando a melhoria da qualidade e da cobertura da notificação.

Neste estudo, com base nos dados obtidos por meio do FormSUS (http://formsus.datasus. gov.br/site/default.php), a fim de acompanhar as ações da RENAST, e dos registros de notificação dos acidentes de trabalho graves e acidentes de trabalho com exposição a material biológico do SINAN, analisam-se fatores associados ao aumento da notificação desses acidentes separadamente.

\section{Métodos}

Este estudo tem como unidades de observação os CEREST estaduais e municipais/regionais de todo o país, sendo a população de referência todos os trabalhadores ativos residentes nos municípios das áreas de abrangência dos Centros. Para selecionar variáveis para o monitoramento destes, definiu-se um modelo lógico com três dimensões potencialmente preditoras: infraestrutura, gestão e organização dos serviços (GEST-ORG); nível de desenvolvimento das ações previstas de vigilância à saúde do trabalhador (NÍVEL-AÇÕES); nível de implantação de atividades relacionadas especificamente à notificação dos acidentes de trabalho (NÍVEL-NOT). Cada uma dessas dimensões foi detalhada em subdimensões, apresentadas na Tabela 1, que correspondem, uma a uma, a variáveis específicas, detalhadas a seguir. A variável resposta foi o aumento proporcional percentual das notificações de acidentes de trabalho graves e acidentes de trabalho com exposição a material biológico, calculada, separadamente, para o período entre 2008 e 2009. As fontes de informação foram o Departamento de Informática do SUS (DATASUS, http://www.datasus.gov. br), dados do FormSUS/2009 e do SINAN/2008 e 2009, respectivamente.

O FormSUS compreende formulários eletrônicos para preenchimento em linha por unidades de saúde. Para o monitoramento da RENAST, dois questionários, denominados de módulos, foram elaborados para a coleta de dados sobre 
Dimensões e subdimensões utilizadas para construir as variáveis do estudo, suas categorias e escores.

\begin{tabular}{|c|c|c|}
\hline Dimensões/Subdimensões & Escore ponderado & Categorias das subdimensões e seus pesos \\
\hline GEST-ORG & $27-76$ & \\
\hline Tempo de funcionamento do CEREST & 12 & Depois de 2003 (4), em 2003 (8), antes de 2003 (12) \\
\hline Excelentes/Boas instalações físicas & 10 & Péssima (2), ruim (4), regular (6), boa (8), excelente (10) \\
\hline Possui equipe mínima & 10 & Não (5), sim (10) \\
\hline Equipe compatível com a demanda & 20 & Péssima (4), ruim (8), regular (12), boa (16), excelente (20) \\
\hline Possui conselho gestor & 8 & Não (4), $\operatorname{sim}(8)$ \\
\hline Possui CIST estadual & 8 & Não (4), sim (8) \\
\hline Possui CIST municipal & 8 & Não (4), sim (8) \\
\hline NÍVEL-AÇÕES & $28-160$ & \\
\hline Vigilância em ambientes de trabalho & 24 & $\begin{array}{l}\text { Não previsto (4), previsto (8), em planejamento (12), em implantação (16), } \\
\text { em funcionamento (20), em funcionamento pleno (24) }\end{array}$ \\
\hline Atende demandas externas * & 10 & Nunca (2), raramente (4), às vezes (6), regularmente (8), sempre (10) \\
\hline Atende demandas de sindicatos & 10 & Nunca (2), raramente (4), às vezes (6), regularmente (8), sempre (10) \\
\hline Atende demandas da mídia & 10 & Nunca (2), raramente (4), às vezes (6), regularmente (8), sempre (10) \\
\hline Participação de trabalhadores & 10 & Nunca (2), raramente (4), às vezes (6), regularmente (8), sempre (10) \\
\hline Rede sentinela estruturada & 24 & $\begin{array}{l}\text { Não previsto (4), previsto (8), em planejamento (12), em implantação (16), } \\
\text { em funcionamento (20), em funcionamento pleno (24) }\end{array}$ \\
\hline Capacitação dos profissionais da rede sentinela & 24 & $\begin{array}{l}\text { Não previsto (4), previsto (8), em planejamento (12), em implantação (16), } \\
\text { em funcionamento (20), em funcionamento pleno (24) }\end{array}$ \\
\hline Estima mortalidade & 24 & $\begin{array}{l}\text { Não previsto (4), previsto (8), em planejamento (12), em implantação (16), } \\
\text { em funcionamento (20), em funcionamento pleno (24) }\end{array}$ \\
\hline Estima morbidade & 24 & $\begin{array}{l}\text { Não previsto (4), previsto (8), em planejamento (12), em implantação (16), } \\
\text { em funcionamento (20), em funcionamento pleno (24) }\end{array}$ \\
\hline NÍVEL-NOT & $32-192$ & \\
\hline Dos acidentes de trabalho fatais & 48 & $\begin{array}{l}\text { Não previsto (8), previsto (16), em planejamento (24), em implantação } \\
\text { (32), em funcionamento (40), em funcionamento pleno (48) }\end{array}$ \\
\hline $\begin{array}{l}\text { Dos acidentes de trabalho graves (com } \\
\text { mutilações) }\end{array}$ & 48 & $\begin{array}{l}\text { Não previsto (8), previsto (16), em planejamento (24), em implantação } \\
\text { (32), em funcionamento (40), em funcionamento pleno (48) }\end{array}$ \\
\hline $\begin{array}{l}\text { Dos acidentes de trabalho graves (com crianças } \\
\text { e adolescentes) }\end{array}$ & 48 & $\begin{array}{l}\text { Não previsto (8), previsto (16), em planejamento (24), em implantação } \\
\text { (32), em funcionamento (40), em funcionamento pleno (48) }\end{array}$ \\
\hline $\begin{array}{l}\text { Dos acidentes de trabalho com exposição a } \\
\text { material biológico }\end{array}$ & 48 & $\begin{array}{l}\text { Não previsto (8), previsto (16), em planejamento (24), em implantação } \\
\text { (32), em funcionamento (40), em funcionamento pleno (48) }\end{array}$ \\
\hline
\end{tabular}

CEREST: Centros de Referência em Saúde do Trabalhador; CIST: Comissão Intersetorial de Saúde do Trabalhador; GEST-ORG: infraestrutura, gestão e organização dos serviços; NÍVEL-AÇÕES: nível de desenvolvimento das ações previstas da vigilância à saúde do trabalhador; NÍVEL-NOT: nível de implantação de atividades relacionadas, especificamente, à notificação dos acidentes de trabalho.

* Ministério Público, comunidade, empresas e programas especiais.

a gestão e implantação das ações previstas no âmbito dos CEREST. O módulo 1 abrange dados sobre a unidade, como o tempo de funcionamento, a infraestrutura, mecanismos voltados à participação dos trabalhadores, dentre outros. $\mathrm{O}$ módulo 2 abarca informações sobre o nível de implementação de ações da vigilância à saúde do trabalhador, relações intra e intersetoriais, estendendo-se do nível de planejamento ao de pleno funcionamento (Tabela 1), dentre outras.

A coleta de dados no FormSUS foi coordenada pela CGSAT, que elaborou os questionários em parceria com a equipe do Centro Colaborador Universidade Federal da Bahia em Vigilância dos Acidentes de Trabalho. Os dados foram preenchidos com base na situação dos CEREST em dezembro de 2008. Em um primeiro contato por correio eletrônico, foram apresentados os objetivos do monitoramento da RENAST por meio do FormSUS, sendo solicitado o preenchimento em linha, na Internet; quando não havia resposta, realizava-se contato telefônico. Para evitar distorções ou parcialidade, recomendou-se que o preenchimento dos questionários traduzisse 
consensos alcançados em reuniões técnicas com a equipe dos CEREST, ou, se isso não fosse possível, por um técnico experiente e familiarizado com o conjunto das ações desenvolvidas pelos Centros.

Por sua vez, o SINAN é um sistema permanente de coleta, transmissão e disseminação de informação sobre doenças e agravos de notificação compulsória, constituindo-se na ferramenta fundamental do sistema de vigilância epidemiológica. Dados são coletados e digitados no nível municipal, transportados para as instâncias regionais de saúde, e encaminhados semanalmente para as secretarias estaduais. Quinzenalmente os dados são enviados para o nível federal do SUS. Para a notificação de doenças e agravos relacionados ao trabalho, as rotinas e fluxos dos dados são definidos com base nos recursos e capacidade operacional dos CEREST e suas respectivas unidades sentinelas.

Unidades sentinelas ou CEREST podem dispor de pessoal capacitado para a digitação no sistema SINAN-NET, sistema de registro em linha, e para o acompanhamento oportuno dos casos, a fim de que estes sejam investigados e se adotem medidas de prevenção ou remediação. Em algumas unidades sentinelas, é realizado apenas o preenchimento das fichas, que são encaminhadas para os CEREST, quando dispõem do acesso ao SINAN-NET, ou diretamente para as secretarias municipais de saúde. As bases de dados do SINAN foram cedidas pela CGSAT, para os anos do estudo.

As variáveis preditoras correspondem às supracitadas três dimensões de análise e suas subdimensões, mostradas em detalhe na Tabela 1 . A GEST-ORG é entendida como aspectos relativos aos recursos institucionais disponíveis, ao tempo e condições de funcionamento e à administração do serviço. A dimensão NÍVEL-AÇÕES traduz o grau de implantação do conjunto de procedimentos esperados, no âmbito da atuação dos CEREST, para a vigilância em saúde do trabalhador, enquanto o NÍVEL-NOT corresponde ao nível de implantação de ações específicas de notificação, como a organização dos dados, a análise, interpretação, dentre outras.

As subdimensões selecionadas do FormSUS para esta análise foram, para a GEST-ORG: tempo de funcionamento; adequação das instalações físicas; disponibilidade de equipe mínima (Portaria $n^{\circ}$. GM/MS 2.437/2005); percepção de que a equipe é compatível com a demanda; existência de conselho gestor para o CEREST; existência de Comissão Intersetorial de Saúde do Trabalhador (CIST), em nível estadual; existência de CIST municipal. A dimensão NÍVEL-AÇÕES compreende as subdimensões: ações de vigilância; atendimento às demandas externas para ações de vigilância - sindicatos e mídia, separadamente; participação de trabalhadores nas ações de vigilância; disponibilidade de rede de unidades sentinelas estruturada; capacitação de profissionais da rede de unidades sentinelas já realizada; dados de mortalidade produzidos e analisados; produção e análise de dados de morbidade e mortalidade. Na dimensão NIVEL-NOT, as subdimensões são referentes à implantação das atividades relacionadas à notificação de acidentes de trabalho graves. Na Tabela 1, apresentam-se as dimensões, suas subdimensões respectivas e faixa de variação dos escores já ponderados.

O grau de implantação de cada dimensão foi mensurado por escores que correspondem à soma dos pontos de cada resposta dada para as respectivas subdimensões. Pesos foram atribuídos a cada uma destas, com base na plausibilidade do papel de cada uma para o desfecho (Tabela 1). Os escores globais de cada dimensão foram categorizados utilizando-se pontos de corte definidos conforme os quartis da faixa de variação possível, sendo, especificamente, implantação avançada ( $>75 \%$ a $100 \%$ ); implantação intermediária (> 50\% a 75\%); implantação incipiente ( $>25 \%$ a $50 \%)$ e não implantado ( $0 \%$ a $25 \%)$. Um escore para avaliação global da implantação foi calculado somando-se os escores de cada uma das três dimensões, categorizado com os mesmos critérios acima descritos.

A variável de desfecho foi a estimativa da variação proporcional percentual (VPP) do número de casos de acidentes de trabalho graves e acidentes de trabalho com exposição a material biológico, definidos de acordo com as normas do SINAN, calculada pela diferença entre o número de acidentes de trabalho notificados no Sistema entre os anos 2008 e 2009, dividida pelo número de notificações no ano de referência inicial, multiplicada por 100. Embora se dispusesse de dados de 2007, sua utilização não foi possível por causa do grande número de valores nulos ou muito pequenos, que expressava o início da implantação do SINAN para acidentes de trabalho. A distribuição da VPP foi transformada em logaritmo para a análise, em virtude da não normalidade da sua distribuição.

A análise foi realizada separadamente para CEREST estaduais e regionais/municipais. Mapas foram elaborados utilizando-se o aplicativo Corel (Corel Corp., Ottawa, Canadá), e fatores associados foram identificados com regressão linear múltipla de mínimos quadrados. A inferência estatística se baseou no teste F, adotando-se um alfa $=0,10$, em face do pequeno tamanho da população do estudo. A modelagem foi do tipo "para trás”, iniciando-se com equações contendo todas 
as variáveis preditoras. Inicialmente, analisaramse as subdimensões de cada dimensão, visando a selecioná-las para a modelagem conjunta de todas as dimensões em uma etapa final. Não foi possível analisar, simultaneamente, todas as subdimensões das três dimensões por causa do pequeno número de unidades de observação. Ademais, nas análises específicas, excluíram-se CEREST que não informaram casos de acidentes de trabalho graves ou acidentes de trabalho com exposição a material biológico em algum dos anos do estudo. Utilizou-se o programa SAS versão 9.1 (SAS Inst., Cary, Estados Unidos) para montagem e preparação das bases de dados. Valores aberrantes foram substituídos pelo valor correspondente a $1 \%$ da distribuição, em ambas as direções. Este estudo não foi conduzido com seres humanos, mas foi aprovado pelo Comitê de Ética em Pesquisa do Instituto de Saúde Coletiva da Universidade Federal da Bahia.

\section{Resultados}

Dos 178 CEREST existentes no final de 2008, 128 $(71,9 \%)$ responderam completa ou parcialmente aos questionários do FormSUS, em referência à situação naquele ano. Em Sergipe, o modo de estruturação da RENAST prescinde do Centro estadual, por isso este não foi incluído na análise que considerou os CEREST estaduais. Dos 128, três $(2,3 \%)$ foram descartados por dados incompletos, especificamente, o CEREST estadual de São Paulo, do Maranhão e do Tocantins, habilitandose 125 para o estudo: 23 estaduais, 100 regionais e dois municipais.

Com relação aos acidentes de trabalho graves, foram 29.629 notificações em 2008 e 30.572 em 2009 , crescimento de $3,2 \%$, enquanto, para os acidentes de trabalho com exposição a material biológico, foram 20.418 e 23.387 , respectivamente, correspondendo a uma elevação de $14,5 \%$ no período.

\section{Implantação dos CEREST}

Em finais de 2008, a implantação dos CEREST estaduais se encontrava ainda por se consolidar (Figuras 1, 2, 3, 4 e 5). Na Figura 1, verifica-se que os CEREST com GEST-ORG mais avançadas se concentravam nas regiões Nordeste, Norte e Centro-oeste; os com menor nível de implantação foram Pernambuco e Santa Catarina. Para a dimensão NÍVEL-AÇÕES, apenas um estado, o Amapá, obteve escore relativo à situação avançada de implantação, enquanto os que ainda necessitavam de expressivos avanços foram Amazonas, Acre, Ceará, Goiás, Pará, Pernambuco, Rio
Grande do Norte, Rondônia e Roraima (Figura 2). Para a última dimensão de análise, NÍVELNOT, observou-se que implantação avançada foi estimada para dez Unidades da Federação (UF): Amapá, Bahia, Distrito Federal, Goiás, Mato Grosso, Mato Grosso do Sul, Minas Gerais, Paraná, Rio Grande do Sul e Roraima (Figura 3); por sua vez, os de implantação menos avançada foram Alagoas, Ceará, Pernambuco, Rio Grande do Norte, Rondônia e Santa Catarina.

A Figura 4 revela que a maior parte $(n=16$; 69,6\%) dos CEREST estaduais se encontrava em etapa intermediária de implantação da GESTORG, enquanto apenas $21,7 \%(n=5)$ foram considerados em etapa avançada. No que se refere à dimensão NÍVEL-AÇÕES, a etapa intermediária ( $n=13 ; 56,5 \%$ ) foi também a mais comum, ficando esta dimensão com a menor proporção de CEREST em etapa avançada, apenas um. Distintamente, para a dimensão NÍVEL-NOT, a maior proporção foi de UF com implantação avançada, $43,5 \%(n=10)$. Entre os CEREST regionais, o padrão de distribuição foi semelhante. A etapa mais prevalente foi a intermediária, para GESTORG ( $\mathrm{n}=55 ; 53,9 \%$ ) e para NÍVEL-AÇÕES ( $\mathrm{n}=$ 51; 50\%), enquanto, para NÍVEL-NOT, a etapa avançada foi a mais frequente, com 57 CEREST, correspondendo a 55,9\% (Figura 5).

A avaliação global, que considerou os escores obtidos em todas as dimensões de análise, revelou que, dentre os CEREST estaduais, três (13\%) apresentaram nível avançado de implantação Amapá, Bahia e Mato Grosso do Sul; 15 (65,2\%), nível intermediário; quatro $(17,4 \%)$ foram classificados no nível incipiente; e um foi identificado como não implantado, especificamente, o CEREST do Estado de Pernambuco. Para os regionais, 26 (25,5\%) apresentaram implantação em nível avançado, 63 (61,8\%) em nível intermediário, e 13 (12,8\%), incipiente, não havendo CEREST identificados como não implantados.

Com os dados de notificação do SINAN nas UF, entre 2008 e 2009 (Figuras 6 e 7), observa-se que, para acidentes de trabalho graves, a maioria ( $\mathrm{n}=17 ; 73,9 \%$ ) apresentou crescimento da notificação, caindo, todavia, em seis UF: Alagoas, Distrito Federal, Goiás, Paraíba, Paraná e Rio de Janeiro. A situação para acidentes de trabalho com exposição a material biológico foi melhor, com crescimento das notificações, chegando a $82,6 \%$ ( $n=19$ ), embora tenha ocorrido queda nos Estados do Amazonas, Ceará, Paraná e Santa Catarina. No nível regional, foi também maioria o crescimento das notificações para ambos os tipos de acidentes de trabalho, porém houve maior proporção de CEREST com queda no período, 40\%, para acidentes de trabalho graves, e $37,4 \%$, para exposição a material biológico. 
Nivel de implantação dos Centros de Referência em Saúde do Trabalhador (CEREST) nas Unidades da Federação, de acordo com a dimensão GEST-ORG (infraestrutura, gestão e organização dos serviços). Brasil, 2008.

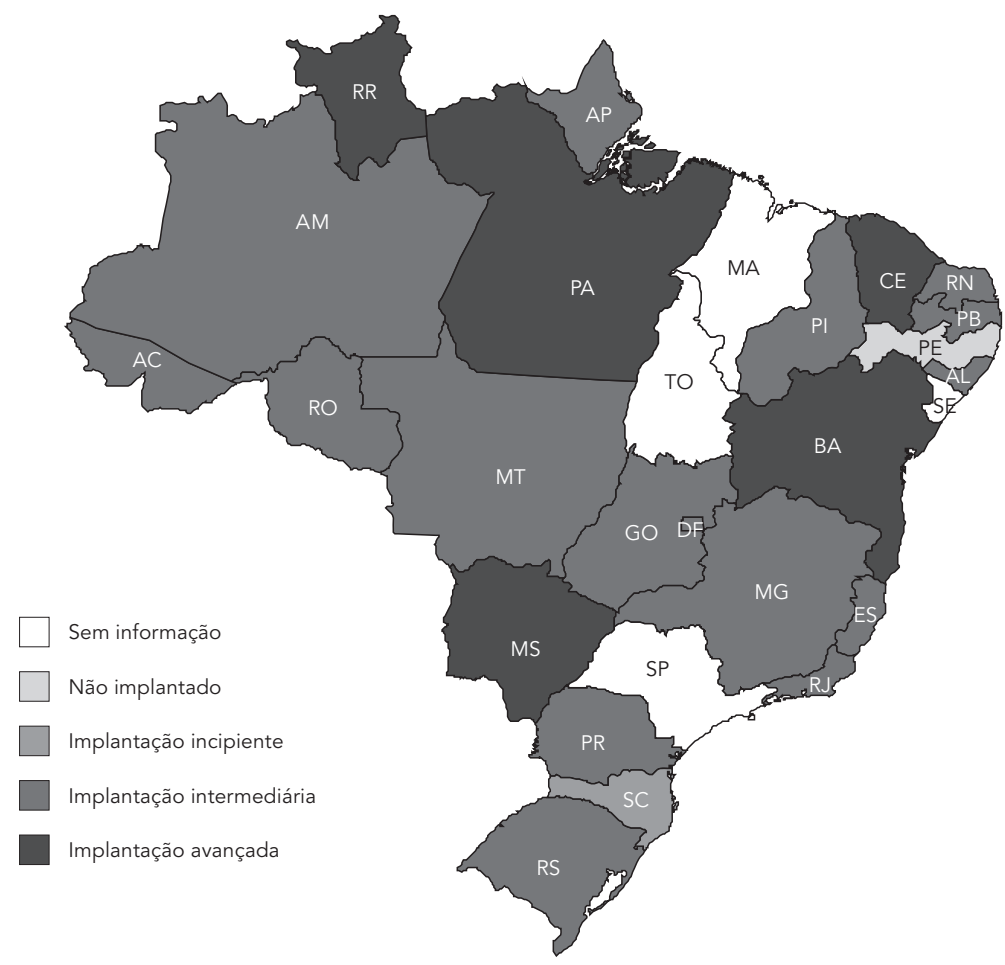

AC: Acre; AL: Alagoas; AM: Amazonas; AP: Amapá; BA: Bahia; CE: Ceará; DF: Distrito Federal; ES: Espírito Santo; GO: Goiás; MA: Maranhão; MG: Minas Gerais; MS: Mato Grosso do Sul; MT: Mato Grosso; PA: Pará; PB: Paraíba; PE: Pernambuco; PI: Piauí; PR: Paraná; RJ: Rio de Janeiro; RN: Rio Grande do Norte; RO: Rondônia; RR: Roraima; RS: Rio Grande do Sul; SC: Santa Catarina; SE: Sergipe; SP: São Paulo; TO: Tocantins.

Fatores associados com a variação da notificação

As dimensões relativas à implantação dos CEREST estaduais, analisadas por indicadores compostos, não se associaram com a variação relativa da notificação de acidentes de trabalho graves ou exposição a material biológico (Tabela 2). Quando se analisou cada uma dessas dimensões por suas respectivas subdimensões, observou-se que, para acidentes de trabalho graves, apenas ter equipe compatível com a demanda, da dimensão GEST-ORG, associouse com o crescimento da notificação ( $\beta=0,27$; $\mathrm{p}<0,10)$. Não houve outras associações estatisticamente significantes. Para os acidentes de trabalho com exposição a material biológico, também nenhuma dimensão analisada se as- sociou com a VPP no período, mas, em relação às subdimensões, dispor de adequada infraestrutura física predizia aumento da VPP $(\beta=0,41$; $\mathrm{p}<0,05)$.

Quando se analisaram os CEREST regionais (Tabela 3), não se verificou, novamente, associação das dimensões com a VPP, seja de acidentes de trabalho graves, seja de acidentes de trabalho com exposição a material biológico. Quanto às subdimensões, para acidentes de trabalho graves, além da equipe compatível com a demanda $(\beta=0,23 ; p<0,10)$, o atendimento pelo CEREST a demandas da mídia $(\beta=0,42$; $\mathrm{p}<0,10$ ) favoreceu o aumento da notificação. Considerando-se os acidentes com exposição a material biológico, observou-se que o escore global da dimensão que corresponde à GESTORG $(\beta=0,09 ; \mathrm{p}<0,05)$ contribuiu para a no- 
Nível de implantação dos Centros de Referência em Saúde do Trabalhador (CEREST) nas Unidades da Federação, de acordo com a dimensão NÍVEL-AÇÕES (nível de desenvolvimento das ações previstas de vigilância à saúde do trabalhador).

Brasil, 2008.

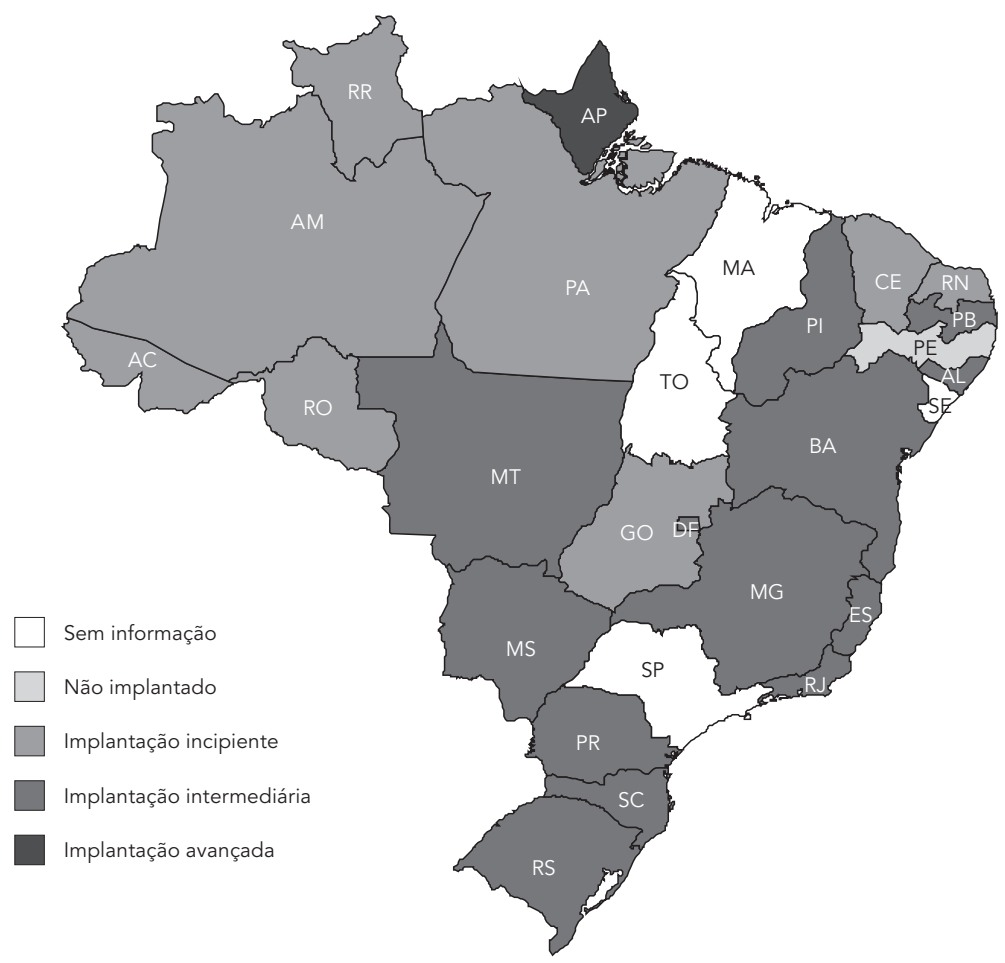

AC: Acre; AL: Alagoas; AM: Amazonas; AP: Amapá; BA: Bahia; CE: Ceará; DF: Distrito Federal; ES: Espírito Santo; GO: Goiás; MA: Maranhão; MG: Minas Gerais; MS: Mato Grosso do Sul; MT: Mato Grosso; PA: Pará; PB: Paraíba; PE: Pernambuco; PI: Piauí; PR: Paraná; RJ: Rio de Janeiro; RN: Rio Grande do Norte; RO: Rondônia; RR: Roraima; RS: Rio Grande do Sul; SC: Santa Catarina; SE: Sergipe; SP: São Paulo; TO: Tocantins.

tificação; para as subdimensões, a contribuição foi apenas de atendimento a demandas da mídia $(\beta=0,56 ; \mathrm{p}<0,10)$ e de capacitação dos profissionais da rede sentinela $(\beta=0,17 ; \mathrm{p}<0,10)$, ambas da dimensão NÍVEL-AÇÕES.

\section{Discussão}

Este estudo demonstra a expansão do número de CEREST no SUS, desde a criação da RENAST em 2002, os quais passaram a abranger todas as UF do país, alcançando 178 CEREST em 2008, um pouco abaixo da meta definida para 2007, que era de duzentas unidades implantadas ${ }^{8}$. Com os dados coletados dos próprios Centros, para monitoramento da sua implantação e avaliação, verificou-se que ainda são necessários esforços visando à sua completa implantação. A maioria dos CEREST prestou informações condizentes com um grau intermediário de implantação de suas instalações físicas, capacidade de gestão e organização dos serviços, o mesmo ocorrendo para o nível de implantação das ações previstas para execução da vigilância à saúde do trabalhador. A dimensão estimada como de maior grau de implantação foi a relativa às ações de notificação dos acidentes de trabalho. Em sua maioria, CEREST estaduais e regionais prestaram informações compatíveis com alto grau de implantação dessas ações. Dentre os fatores que impulsionaram o crescimento da notificação de acidentes de trabalho nos CEREST estaduais, destacaramse a capacidade instalada e a dotação de pessoal, expressos pelo papel da equipe compatível com a demanda, para os acidentes de trabalho graves, e 
Nível de implantação dos Centros de Referência em Saúde do Trabalhador (CEREST) nas Unidades da Federação, de acordo com a dimensão NíVEL-NOT (nível de implantação das atividades de notificação dos acidentes de trabalho). Brasil, 2008.

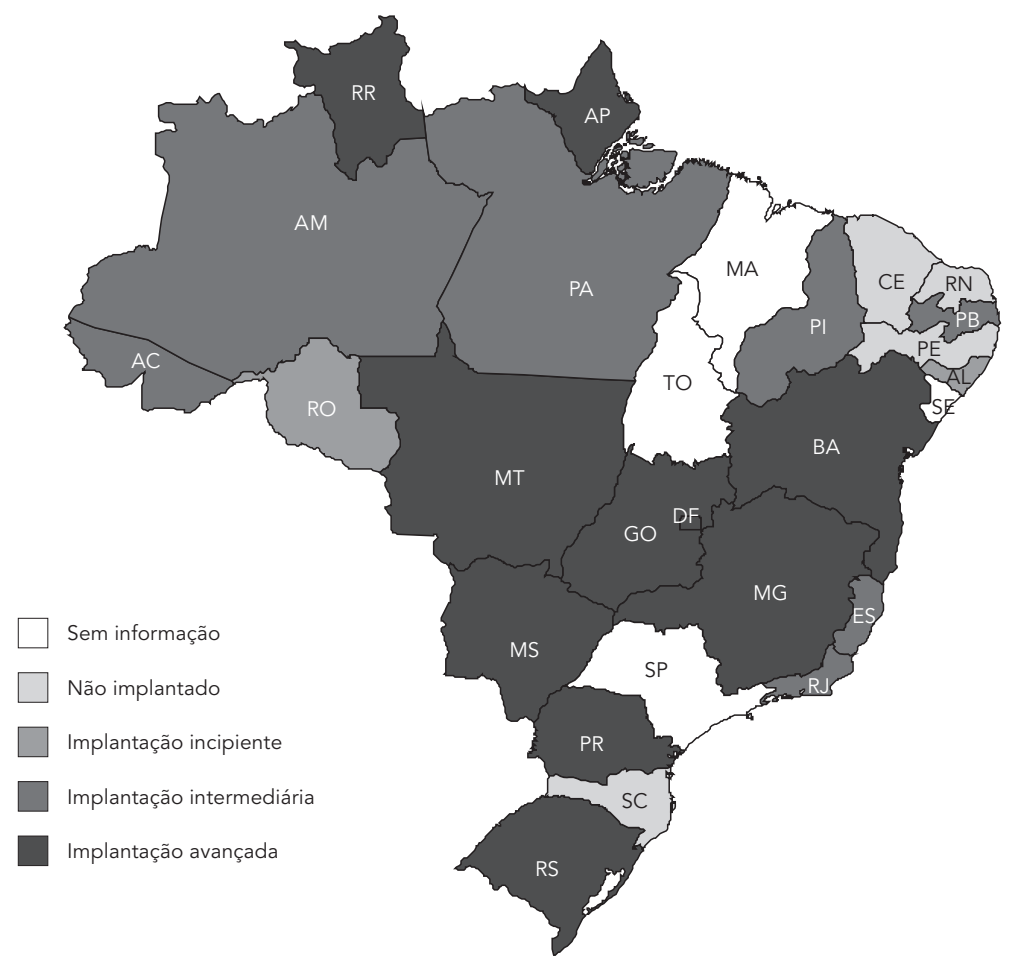

AC: Acre; AL: Alagoas; AM: Amazonas; AP: Amapá; BA: Bahia; CE: Ceará; DF: Distrito Federal; ES: Espírito Santo; GO: Goiás; MA: Maranhão; MG: Minas Gerais; MS: Mato Grosso do Sul; MT: Mato Grosso; PA: Pará; PB: Paraíba; PE: Pernambuco; PI: Piauí; PR: Paraná; RJ: Rio de Janeiro; RN: Rio Grande do Norte; RO: Rondônia; RR: Roraima; RS: Rio Grande do Sul; SC: Santa Catarina; SE: Sergipe; SP: São Paulo; TO: Tocantins.

pela adequada infraestrutura física, para acidentes de trabalho com exposição a material biológico. Entre os CEREST regionais, além de equipe compatível com a demanda, apareceram como propulsores as ações que denotam dinamismo das relações dos CEREST com a sociedade, especificamente no atendimento de demandas externas, como a da mídia.

Ainda são poucos os estudos sobre a implantação dos CEREST e da RENAST, em especial sobre as dificuldades e perspectivas da gestão em saúde do trabalhador no SUS. Existem indicações de que as dificuldades na implantação das ações em saúde do trabalhador seriam semelhantes àquelas descritas para o SUS, com o agravante das especificidades desse campo 6,9 . Um exemplo seria a falta de articulação entre as instituições responsáveis por ações em saúde do trabalhador, como o Ministério do Trabalho e Emprego, e a Previdência Social, desarticulação que é expressa pela falta de compartilhamento de dados úteis para a vigilância à saúde do trabalhador, especialmente os da notificação, como os provenientes das inspeções de ambientes de trabalho e dos sistemas de benefícios 9. Outro problema seria a separação entre a saúde do trabalhador e as demais vigilâncias desenvolvidas pelo próprio SUS, além dos limites da compreensão do trabalho como determinante da saúde pelos profissionais da saúde 6 .

Em um estudo qualitativo baseado em narrativas de profissionais de saúde dos CEREST, Dias et al. 10 relataram haver dificuldades de compreensão do papel desses serviços, e em especial do que vêm a ser ações de vigilância à saúde do trabalhador, limitada capacitação dos técnicos pa- 
Distribuição dos Centros de Referência em Saúde do Trabalhador (CEREST) estaduais, de acordo com o nível de implantação das dimensões de análise. Brasil, 2008.

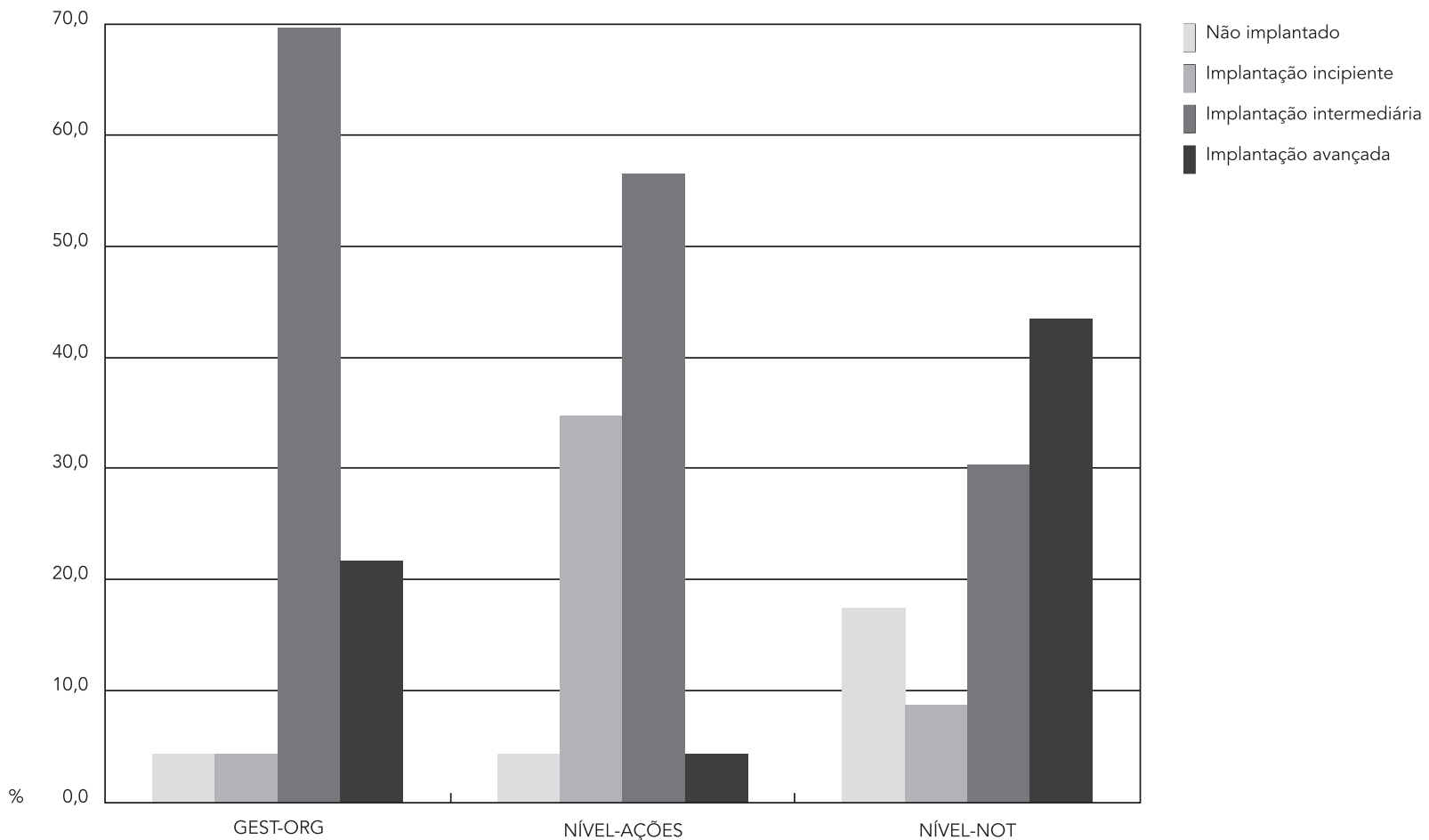

GEST-ORG: infraestrutura, gestão e organização dos serviços; NÍVEL-AÇÕES: nível de desenvolvimento das ações previstas de vigilância à saúde do trabalhador; NÍVEL-NOT: nível de implantação das atividades de notificação dos acidentes de trabalho.

ra estas ações, além da falta de suporte especializado. Os participantes referiram ainda conflitos políticos e de interesse envolvendo governantes locais, comumente donos de empresas, e também profissionais que acumulam vínculos no setor privado e público; ademais, encontraram evidências de dificuldades relativas à estabilidade de emprego e profissionalização. Além disso, há problemas em alcançar o total da região coberta pelos CEREST, para além dos municípiossede, porque a regionalização dos Centros é própria, distinta da regionalização adotada pelo SUS nos estados e municípios 6 . Esses achados são consistentes com os fatores associados à notificação encontrados neste estudo, os quais ressaltam a importância das equipes e da ação intersetorial.

A presente investigação, ao propor analisar os fatores associados à notificação dos acidentes de trabalho graves e exposição a material biológico, não se apoiou apenas na percepção e apreciação das equipes dos CEREST, registradas nas respostas dadas aos instrumentos de monitoramento do FormSUS; também comparou essas informações com os dados de notificação de acidentes de trabalho no SINAN. Estes dados expressam, objetivamente, um produto das ações dos CEREST, principalmente das unidades sentinelas que os apoiam e por eles são apoiadas, em sua própria implantação. Com isso, confrontaram-se percepções com dados objetivos, reduzindo-se os limites inerentes a julgamentos sobre os próprios desempenhos, subjacentes aos dados registrados pelo FormSUS. Ainda assim, algum grau de viés de informação parece ter se refletido e contribuído para algumas contradições observadas: alguns CEREST registraram dados revelando alto grau de implantação das dimensões em análise, mas o seu desempenho na notificação de acidente de trabalho foi, contraditoriamente, pouco expressivo ou de queda. 
Distribuição dos Centros de Referência em Saúde do Trabalhador (CEREST) regionais, de acordo com o nível de implantação das dimensões de análise. Brasil, 2008.

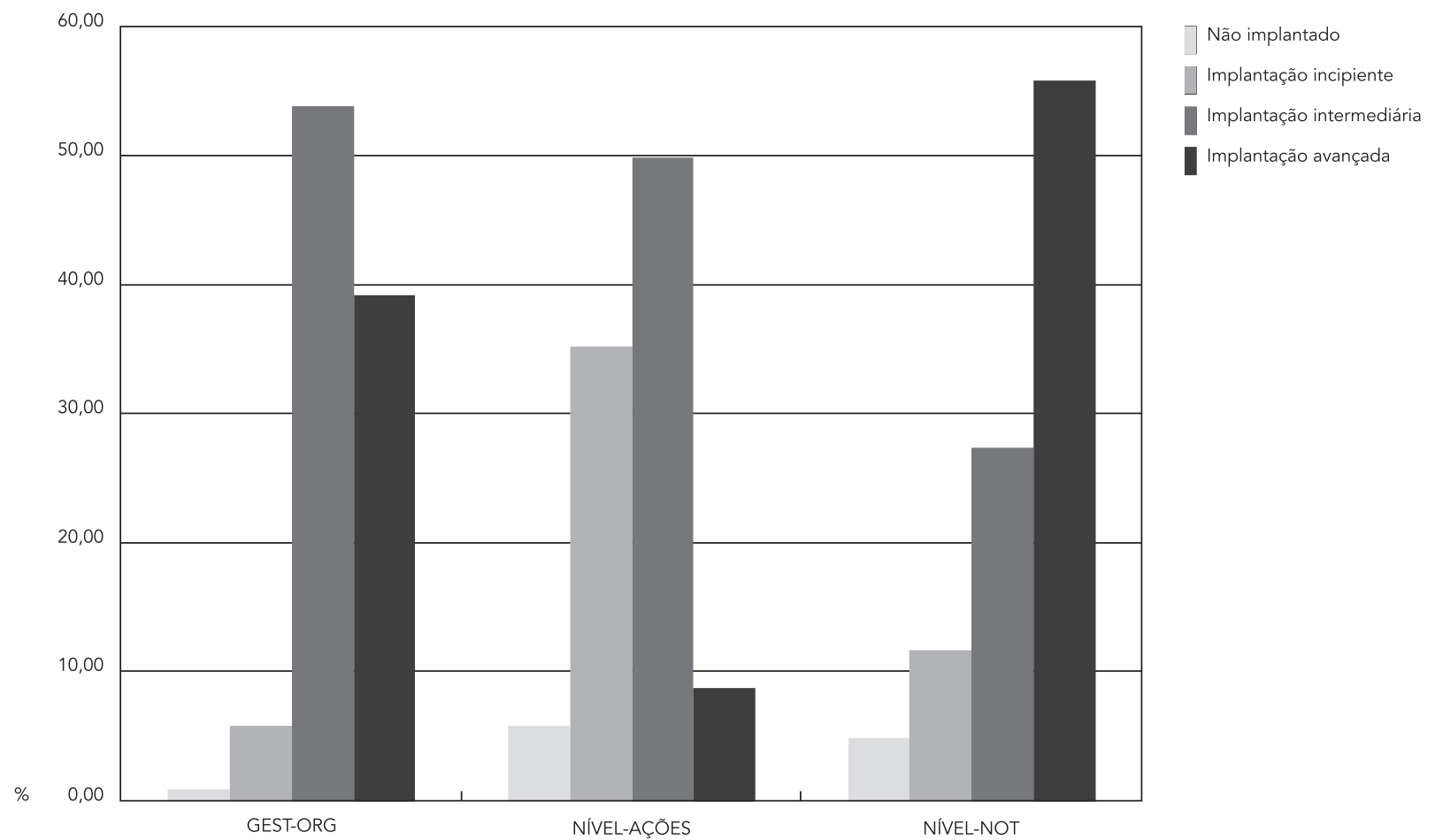

GEST-ORG: infraestrutura, gestão e organização dos serviços; NÍVEL-AÇÕES: nível de desenvolvimento das ações previstas de vigilância à saúde do trabalhador; NÍVEL-NOT: nível de implantação das atividades de notificação dos acidentes de trabalho.

Variáveis relativas à disponibilidade de recursos pelo CEREST e agilidade da execução financeira não foram incluídas pela falta de dados e inconsistências. A análise dos CEREST estaduais ficou comprometida pela ausência de São Paulo, que representa quase $50 \%$ dos trabalhadores do país, e pela presença de sistemas paralelos de notificação que precederam o SINAN, no Rio Grande do Sul e no Rio de Janeiro, que ainda permaneciam ativos. O pequeno poder estatístico do estudo é inerente ao reduzido número de CEREST estaduais e regionais, o que comprometeu a modelagem para as subdimensões.

A distribuição da VPP dos acidentes de trabalho apresentou superdispersão, ou seja, uma grande variação entre os valores observados, especialmente pela presença de valores próximos à unidade, o que foi minimizado pelo tratamento de valores aberrantes e transformação logarítmica. Além disso, a variável desfecho, VPP dos acidentes de trabalho, não mede a subnotificação desses agravos, construto que, de fato, deveria ser empregado, caso fosse factível sua utilização. Assim, VPP pequena pode representar não apenas crescimento reduzido, mas o alcance de patamar máximo, expressando mínima subnotificação. Adicionalmente, o fluxo de informação do SINAN dos níveis locais para os superiores parece ainda estar em consolidação, e os CEREST têm relatado dificuldades operacionais nos procedimentos de notificação, sobretudo quando esta se dá pela Internet, e no preenchimento de alguns campos, como o Cadastro Nacional de Pessoa Jurídica (CNPJ) da empresa, dentre outros.

O crescimento da notificação dos acidentes de trabalho entre 2008 e 2009 foi muito menor do que o ocorrido entre 2007 e 2008, tanto para acidentes de trabalho graves, quanto por acidentes de trabalho com exposição a material biológico, o que demonstra, em relação à tendência do ano anterior ${ }^{3}$, a queda da intensidade e vigor da implantação das ações ligadas à atividade, em- 
Distribuição da variação proporcional percentual (VPP) do número de acidentes de trabalho graves notificados no Sistema de Informações de Agravos de Notificação (SINAN), entre 2008 e 2009, de acordo com as Unidades da Federação.

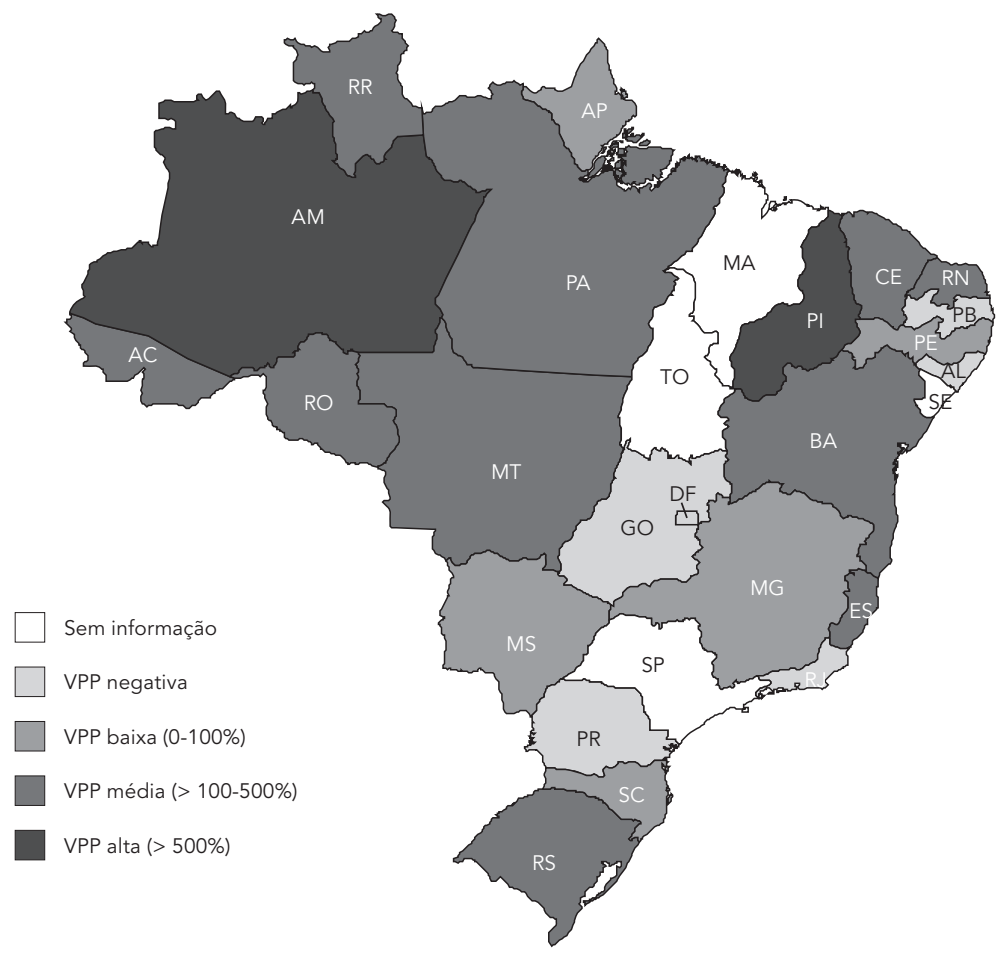

AC: Acre; AL: Alagoas; AM: Amazonas; AP: Amapá; BA: Bahia; CE: Ceará; DF: Distrito Federal; ES: Espírito Santo; GO: Goiás; MA: Maranhão; MG: Minas Gerais; MS: Mato Grosso do Sul; MT: Mato Grosso; PA: Pará; PB: Paraíba; PE: Pernambuco; PI: Piauí; PR: Paraná; RJ: Rio de Janeiro; RN: Rio Grande do Norte; RO: Rondônia; RR: Roraima; RS: Rio Grande do Sul; SC: Santa Catarina; SE: Sergipe; SP: São Paulo; TO: Tocantins.

bora esteja claro que o número de notificações encontra-se muito aquém do esperado.

Notificações e investigações dos acidentes de trabalho estão entre os principais requisitos para as ações de vigilância. Incentivos para a conformação de uma rede com unidades notificantes devem se refletir nas ações de estruturação da rede sentinela, e de capacitação dos seus profissionais. Essas ações favorecem a consolidação das estratégias de implantação do SINAN e a continuidade das atividades desenvolvidas em busca da superação da grande subnotificação de acidentes de trabalho.

Neste trabalho, demonstrou-se a importância e potencialidades dos CEREST na produção de informações na área de saúde do trabalhador; sendo assim, estratégias que favoreçam o aumento das notificações precisam ser desen- volvidas. Uma iniciativa concreta do Ministério da Saúde, para os anos 2010 e 2011, foi o processo de pactuação dos indicadores de saúde do Pacto pela Vida, um dos componentes do Pacto pela Saúde, que já aponta para esta tendência, contemplando indicadores da área de saúde do trabalhador, voltados especificamente para o aumento das notificações. Esse empreendimento certamente irá estimular gestores municipais a se comprometerem com as ações de saúde do trabalhador, e com o papel importante da notificação de agravos relacionados ao trabalho, para o diagnóstico da situação nos territórios de governabilidade. 
Distribuição da variação proporcional percentual (VPP) do número de acidentes de trabalho com exposição a material biológico notificados no Sistema de Informações de Agravos de Notificação (SINAN), entre 2008 e 2009, de acordo com as Unidades da Federação.

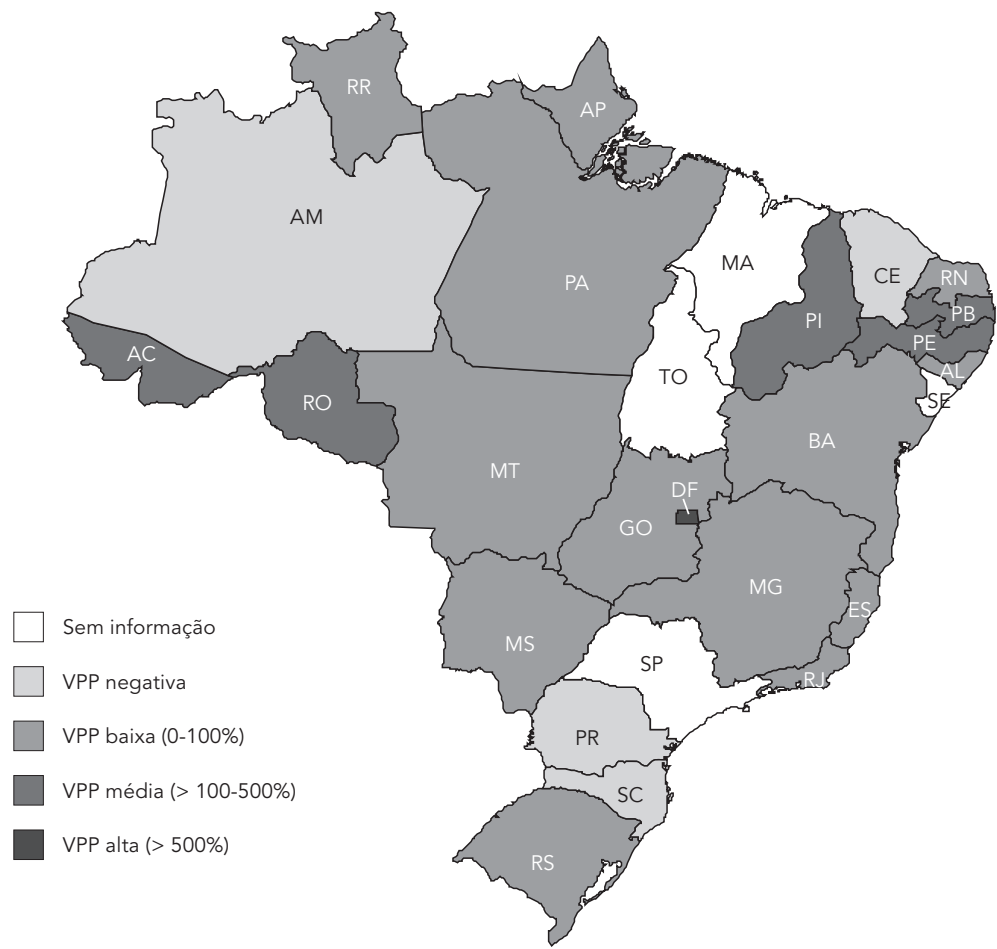

AC: Acre; AL: Alagoas; AM: Amazonas; AP: Amapá; BA: Bahia; CE: Ceará; DF: Distrito Federal; ES: Espírito Santo; GO: Goiás; MA: Maranhão; MG: Minas Gerais; MS: Mato Grosso do Sul; MT: Mato Grosso; PA: Pará; PB: Paraíba; PE: Pernambuco; PI: Piauí; PR: Paraná; RJ: Rio de Janeiro; RN: Rio Grande do Norte; RO: Rondônia; RR: Roraima; RS: Rio Grande do Sul; SC: Santa Catarina; SE: Sergipe; SP: São Paulo; TO: Tocantins. 
Coeficientes da regressão linear para a associação entre variáveis relativas ao nível de implantação dos Centros de Referência em Saúde do Trabalhador (CEREST) estaduais e a variação proporcional das notificações de acidentes de trabalho graves e acidentes de trabalho com exposição a materiais biológicos, entre 2008 e 2009.

Dimensões/Subdimensões
Acidentes graves

( $n=23$ CEREST)

Modelo 1 (apenas uma subdimensão)

$\beta$

\section{Modelo 3 \\ (final)}

Acidentes com exposição a materiais biológicos ( $n=23$ CEREST)

Modelo 1 (apenas uma subdimensão)

$\beta$
Modelo 3

(final)

$\beta$

\begin{tabular}{|c|c|c|c|c|}
\hline \multicolumn{5}{|l|}{ GEST-ORG } \\
\hline Tempo de funcionamento do CEREST & $-0,16$ & 0,04 & $-0,05$ & \\
\hline Excelentes/Boas instalações físicas & 0,16 & & 0,19 & 0,41 * \\
\hline Possui equipe mínima & 0,03 & & 0,04 & \\
\hline Equipe compatível com a demanda & $0,21 \star \star$ & $0,27 \star \star$ & $-0,01$ & \\
\hline Possui conselho gestor & 0,37 & & $-0,33$ & $-0,30$ \\
\hline Possui CIST estadual & $-0,04$ & & $-0,37$ * & $-0,56$ \\
\hline Possui CIST municipal & $0,30 * \star$ & 0,24 & $-0,01$ & \\
\hline \multicolumn{5}{|l|}{ NÍVEL-AÇÕES } \\
\hline Vigilância em ambientes de trabalho & $-0,04$ & & $-0,02$ & \\
\hline Atende demandas externas *** & $-0,31$ & & 0,02 & \\
\hline Atende demandas de sindicatos & $-0,10$ & & 0,08 & 0,09 \\
\hline Atende demandas da mídia & $-0,37 \star \star$ & $-0,31$ & 0,03 & \\
\hline Participação de trabalhadores & $-0,25$ & $-0,18$ & 0,14 & 0,18 \\
\hline Rede sentinela estruturada & $-0,13$ & & $-0,07$ & $-0,11$ \\
\hline Capacitação dos profissionais da rede sentinela & 0,00 & & $-0,05$ & \\
\hline Estima mortalidade & $-0,13$ & $-0,03$ & 0,00 & \\
\hline Estima morbidade & $-0,03$ & & 0,04 & \\
\hline \multicolumn{5}{|l|}{ NÍVEL-NOT } \\
\hline Dos acidentes de trabalho fatais & $-0,06$ & $-0,47$ & 0,03 & $-0,07$ \\
\hline Dos acidentes de trabalho graves (com mutilações) & $-0,05$ & 0,40 & 0,02 & \\
\hline $\begin{array}{l}\text { Dos acidentes de trabalho graves (com crianças e } \\
\text { adolescentes) }\end{array}$ & $-0,05$ & 0,02 & 0,03 & 0,02 \\
\hline $\begin{array}{l}\text { Dos acidentes de trabalho com exposição a material } \\
\text { biológico }\end{array}$ & $-0,03$ & & 0,04 & 0,07 \\
\hline
\end{tabular}

CIST: Comissão Intersetorial de Saúde do Trabalhador; GEST-ORG: infraestrutura, gestão e organização dos serviços; NIVEL-AÇÕES: nível de desenvolvimento das ações previstas da vigilância à saúde do trabalhador; NíVEL-NOT: nível de implantação de atividades relacionadas, especificamente, à notificação dos acidentes de trabalho.

* Valor de $\mathrm{p}<0,05$;

** Valor de $\mathrm{p}<0,10$;

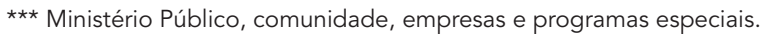


Coeficientes da regressão linear para a associação entre variáveis relativas ao nível de implantação dos Centros de Referência em Saúde do Trabalhador (CEREST) regionais e a variação proporcional das notificações de acidentes de trabalho graves e acidentes de trabalho com exposição a materiais biológicos, entre 2008 e 2009.

\section{Dimensões/Subdimensões}

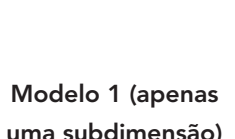

$\beta$

\section{Acidentes graves \\ ( $\mathrm{n}=80$ CEREST)}

Modelo 2 (todas as subdimensões)
Tempo de funcionamento do CEREST

Excelentes/Boas instalações

físicas

Possui equipe mínima

Equipe compatível com a

demanda

Possui conselho gestor

Possui CIST estadual

Possui CIST municipal

NÍVEL-AÇÕES

Vigilância em ambientes de trabalho

Atende demandas externas ***

Atende demandas de

sindicatos

Atende demandas da mídia

Participação de trabalhadores

Rede sentinela estruturada

Capacitação dos profissionais

da rede sentinela

Estima mortalidade

Estima morbidade

\section{NÍVEL-NOT}

Dos acidentes de trabalho

fatais

Dos acidentes de trabalho

graves (com mutilações)

Dos acidentes de trabalho

graves (com crianças e

adolescentes)

Dos acidentes de trabalho com exposição a material biológico

0,08

0,00

$-0,03$

$-0,04$

0,02

0,01

0,02

\section{$\beta$}

$-0,01$

0,33

0,09

0,10

0,12

0,14

( $n=91$ CEREST)

Modelo 3

(final)

Modelo 2 (todas as Modelo

$\beta$ subdimensões) 3 (final)

$\beta$

\begin{tabular}{|c|c|c|c|c|c|}
\hline 0,06 & $-0,15$ & & 0,15 & $-0,04$ & \\
\hline 0,20 ** & 0,27 ** & 0,23 ** & 0,25 * & 0,19 & 0,15 \\
\hline$-0,25$ & $-0,23$ & $-0,22$ & $0,37 * \star$ & 0,25 & 0,29 \\
\hline 0,19 & 0,26 & 0,24 & 0,01 * & 0,10 & $-0,04$ \\
\hline 0,28 & 0,06 & 0,08 & $-0,02$ & $-0,10$ & \\
\hline
\end{tabular}

$\begin{array}{llllll}-0,04 & -0,04 & -0,04 & -0,02 & -0,41 & -0,06\end{array}$

$\begin{array}{cccccc}-0,14 & -0,77^{*} & -0,75 * * & -0,03 & -0,41 & -0,42 \\ 0,07 & 0,32 & 0,28 & -0,04 & -0,24 & -0,22\end{array}$

0,32

$0,42 * \star$

0,14

0,56 *

0,56 **

0,38 **

0,01

0,02

0,02

0,12 **

0,05

$-0,03$

$-0,01$

0,15 *

0,17 *

0,17 **

$-0,09$

0,01

$-0,08$

0,06

0,05

$-0,10$ **

$-0,05$

0,03

0,03

0,01

0,09

0,03

0,00

0,00

0,00

$-0,03$

0,00

0,03

0,06

0,06

0,05

CIST: Comissão Intersetorial de Saúde do Trabalhador; GEST-ORG: infraestrutura, gestão e organização dos serviços; NÍVEL-AÇÕES: nível de desenvolvimento das ações previstas da vigilância à saúde do trabalhador; NíVEL-NOT: nível de implantação de atividades relacionadas, especificamente, à notificação dos acidentes de trabalho.

* Valor de $\mathrm{p}<0,05$;

** Valor de $\mathrm{p}<0,10$;

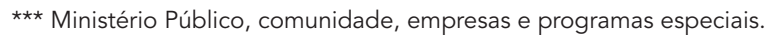




\section{Resumo}

Analisa-se como os Centros de Referência em Saúde do Trabalhador (CEREST) contribuem para as notificações de acidentes de trabalho graves e daqueles com exposição a material biológico, no Sistema de Informações de Agravos de Notificação (SINAN), Sistema Único de Saúde (SUS). Foram empregados dados do FormSUS e do SINAN, agregados para as áreas de cobertura dos CEREST. Foram obtidos dados válidos de 125 CEREST, 23 estaduais e 102 regionais. A maioria dos CEREST foi considerada implantada. O aumento da notificação de acidentes de trabalho graves foi maior quando a equipe era compativel com a demanda e se atendiam a demandas externas e da mídia. Para as exposições a material biológico, CEREST com boas instalações físicas, que atendiam a demandas da mídia e tinham capacitado pessoal da rede sentinela, apresentaram maior aumento da notificação. A infraestrutura, quantidade e capacitação de pessoal, além do atendimento a demandas externas, são importantes para o aumento das notificações e devem ser priorizados para reduzir a expressiva subnotificação dos acidentes de trabalho.

Notificação de Acidentes de Trabalho; Acidentes de Trabalho; Saúde do Trabalhador; Sistemas de Informação

\section{Colaboradores}

A. Galdino, V. S. Santana e S. Ferrite participaram da concepção e projeto, análise e interpretação dos dados, redação do artigo, revisão crítica do conteúdo intelectual e aprovação da versão final para publicação.

\section{Agradecimentos}

À Coordenação Geral de Saúde do Trabalhador (CGSAT) do Ministério da Saúde e do grupo Análise de Situação de Saúde Ambiental e da Saúde do Trabalhador (ASISAST), que disponibilizaram os dados obtidos pelo FormSUS e Sistema de Informações de Agravos de Notificação (SINAN). Agradecimentos especiais ao Dr. Jorge Huet Mesquita Machado e ao Dr. Augusto Campos, pelas contribuições ao longo da elaboração. Agradecemos também às equipes dos Centros de Referência em Saúde do Trabalhador (CEREST) da Rede Nacional de Atenção Integral à Saúde do Trabalhador (RENAST) que pacientemente registraram os dados aqui analisados. À Secretaria da Saúde do Estado da Bahia, Conselho Nacional de Desenvolvimento Científico e Tecnológico (CNPq) e CGSAT/Ministério da Saúde, pelo financiamento. Este trabalho é um dos produtos de Centro Colaborador em Vigilância dos Acidentes de Trabalho, do Instituto de Saúde Coletiva, da Universidade Federal da Bahia e CGSAT.

\section{Referências}

1. Conceição PSA, Nascimento IBO, Oliveira PS, Cerqueira MRM. Acidentes de trabalho atendidos em serviço de emergência. Cad Saúde Pública 2003; 19:111-7.

2. Ministério da Previdência Social. Anuário estatístico da Previdência Social 2008. http://www. previdenciasocial.gov.br/conteudoDinamico. php?id=850 (acessado em 27/Ago/2010).

3. Santana VS, Moura MCP, Soares JFS, Guedes MH. Acidentes de trabalho no Brasil: dados de notificação do SINAN 2007-2008. Salvador: Centro Colaborador Universidade Federal da Bahia/Coordenação Geral da Saúde do Trabalhador, Ministério da Saúde; 2009.

4. Correa PRL, Assunção AA. A subnotificação de mortes por acidentes de trabalho: estudo de três bancos de dados. Epidemiol Serv Saúde 2003; 12:203-12.

5. Cordeiro RC, Sakate M, Clemente APG, Diniz CS, Donalisio MR. Subnotificação de acidentes do trabalho não fatais em Botucatu, SP, 2002 Rev Saúde Pública 2005; 39:254-60.

6. Dias EC, Hoefel MG. O desafio de implementar as ações de saúde do trabalhador no SUS: a estratégia da RENAST. Ciênc Saúde Coletiva 2005; 10:817-27.

7. Santana VS, Silva JM. Os 20 anos da saúde do trabalhador no Sistema Único de Saúde do Brasil: limites, avanços e desafios. In: Departamento de Análise de Situação de Saúde, Secretaria de Vigilância em Saúde, Ministério da Saúde, organizador. Saúde Brasil 2008: 20 anos de Sistema Único de Saúde no Brasil. Brasília: Ministério da Saúde; 2009. p. 175-204. (Série G. Estatística e Informação em Saúde).

8. Brasil. Portaria n ${ }^{\circ}$. 2.437/GM, de 7 de dezembro de 2005. Dispõe sobre a ampliação e o fortalecimento da Rede Nacional de Atenção Integral à Saúde do Trabalhador - RENAST - no Sistema Único de Saúde - SUS. Diário Oficial da União 2005; 9 dez.

9. Santana VS, Dias EC, Franco Netto G, Correa-Filho H, Senna da Silva J, Vasconcelos FD, et al. The occupational health and safety institutional analysis - the case of informal employment in Brazil. Salvador: Instituto de Saúde Coletiva, Universidade Federal da Bahia/London: Women Informal Employment Globalizing and Organizing; 2010.

10. Dias EC, Chiavegatto CV, Silva TL, Reis JC, Silva JM. Construção da RENAST em Minas Gerais: a contribuição dos Centros de Referência em Saúde do Trabalhador (CEREST), 2002-2007. Rev Méd Minas Gerais 2010; 20:66-74.

Recebido em 07/Dez/2010

Versão final reapresentada em 28/Jun/2011

Aprovado em 22/Ago/2011 